\title{
CMEARTICLE
}

\section{A practical clinical approach to liver fibrosis}

Rahul $\underline{K u m a r}^{1}$, MBBS, MRCP, Eng Kiong $\underline{T e O}^{1}$, MBBS MRCP, How Choon $\underline{H o w}^{2,3}$, MMed, FCFP, Teck Yee $\underline{\text { Wong }}^{4}$, MMed, MHPEd, Tiing Leong Ang $^{1}$, FRCPEd, FAMS

Ms Chew, a 35-year-old executive, was found to have fatty liver on her recent pre-employment health checkup. She visited your clinic and showed you her test results. Her liver biochemistry assessment was normal. Ultrasonography of the liver was normal except for mild fatty infiltration of the liver. Her body mass index was 24, and she was generally well. Ms Chew was concerned about her liver health and whether she had liver hardening or fibrosis.

\section{WHAT IS LIVER FIBROSIS?}

Liver fibrosis, regardless of the underlying aetiology, is a consequence of the accumulation of extracellular matrix protein, including collagen, in the liver. It is a dynamic process that reflects imbalanced extracellular matrix turnover. This process is caused by persistent liver damage and consequent wound healing reaction. It can progress to cirrhosis, portal hypertension and hepatocellular carcinoma, leading to increased morbidity and mortality. ${ }^{(1,2)}$

Common causes of liver fibrosis that also lead to chronic liver disease (CLD) are listed in Box 1. Liver fibrosis has an insidious onset, slowly progressing over many years and typically taking over 20 years to develop into liver cirrhosis. Rapid progression over a few years to liver cirrhosis can also occur in some cases. Genetic as well as environmental factors influence the natural history of liver fibrosis. Genetic variations in candidate genes may influence the rate of fibrosis progression, ${ }^{(3)}$ which may explain the broad spectrum of clinical phenotypes with the same aetiological agents. Effectively treating and controlling the aetiology and risk factors of fibrosis progression can potentially reverse fibrosis.

\section{HOW COMMON IS THIS IN MY PRACTICE?}

Liver fibrosis results from persistent liver damage and consequent wound healing reaction due to CLD. In Singapore, chronic hepatitis B (CHB), non-alcoholic fatty liver disease (NAFLD) and alcoholic liver disease are common causes of CLD. Chronic hepatitis $\mathrm{C}(\mathrm{CHC})$ is more prevalent among intravenous drug users. Other aetiological factors that can give rise to CLD are primary biliary cirrhosis, autoimmune hepatitis and haemochromatosis.

The prevalence of CLD and consequent liver fibrosis is challenging to assess. Recently, epidemiological data from an Italian general practitioner database revealed that $37.2 \%$ of patients had either overt or occult CLD based on liver function and imaging modalities. ${ }^{(4)}$ CLD is typically diagnosed on health screening or incidentally, after the investigation of an abnormal liver panel. This is because CLD, or even liver cirrhosis, is often asymptomatic until complications appear. Hence, a high index of suspicion is necessary.

The stages of liver fibrosis in CLD, such as NAFLD, define the patient's overall morbidity and mortality; the higher the stage of fibrosis, the worse the prognosis (Fig. 1). ${ }^{(5)}$ The amount of liver fibrosis is correlated with the risk of developing liver cirrhosis and liver-related complications in viral and non-viral CLD. ${ }^{(6,7)}$ The assessment of liver fibrosis is thus crucial in making therapeutic decisions and predicting outcomes.

\section{WHAT CAN I DO IN MY PRACTICE?}

After establishing the presence of CLD and determining the aetiology, the next step would be to determine whether a patient needs specialised care, including follow-up and treatment. Patients who have $\mathrm{CHB}$ with persistently elevated transaminases

\section{Box 1. Common causes of liver fibrosis:}

- Chronic viral hepatitis B and C

- Non-alcoholic fatty liver disease

- Alcoholic liver disease

- Autoimmune liver diseases

- Iron and copper overload

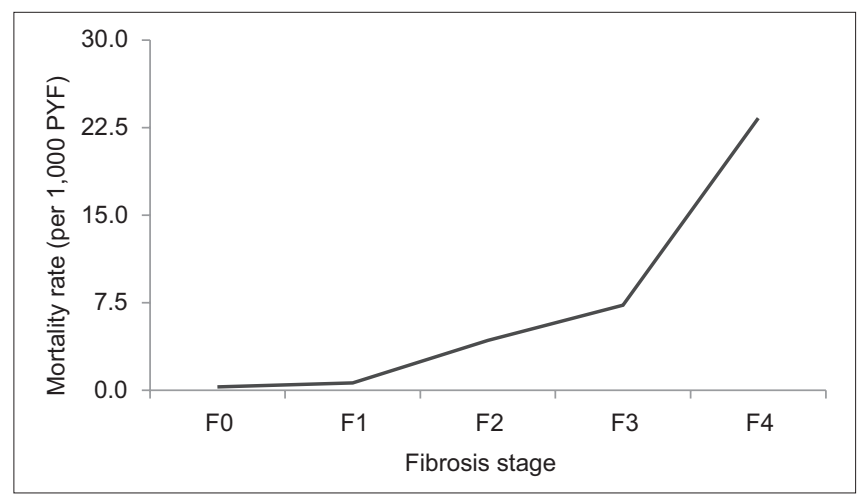

Fig. 1 Fibrosis stage-specific liver-related mortality rate in non-alcoholic fatty liver disease (adapted from Dulai et al). ${ }^{(5)}$ PYF: patient-years of follow-up

${ }^{1}$ Department of Gastroenterology and Hepatology, ${ }^{2}$ Care and Health Integration, Changi General Hospital, ${ }^{3}$ Family Medicine Academic Clinical Programme, SingHealth Duke-NUS Academic Medical Centre, ${ }^{4}$ Department of Continuing and Community Care, Tan Tock Seng Hospital, Singapore

Correspondence: Dr Rahul Kumar, Associate Consultant, Department of Gastroenterology and Hepatology, Changi General Hospital, 2 Simei Street 3 , Singapore 529889. rahul_kumar@cgh.com.sg 
(> $2 \times$ upper limit of normal), patients of any aetiology with advanced fibrosis, and those with unknown aetiology should be referred for specialist care. The fibrosis assessment of patients should divide them into the advanced fibrosis or not-advanced fibrosis groups. ${ }^{(8,9)}$ Noninvasive tests, especially serology-based composite scores, can be used to determine whether patients with mild abnormalities in liver chemical values need referrals to liver specialists. With NAFLD on the rise due to the epidemic of diabetes mellitus and obesity, this becomes even more relevant. ${ }^{(10)}$ Depending on the aetiology, composite scores such as the NAFLD fibrosis score (NFS) and Fibrosis-4 (FIB-4) index for NAFLD; FIB-4 for $\mathrm{CHB}$; and aspartate aminotransferase to platelet ratio index (APRI) score for the hepatitis $\mathrm{C}$ virus (HCV) provide cost-effective and efficient risk stratification when performed in the primary care clinic, identifying patients who have advanced fibrosis or indeterminate scores warranting referral to a specialist. ${ }^{(11,12)}$

Most patients having NAFLD with 'low risk' on the composite scoring systems can be followed up at the primary care clinic. Patients with 'intermediate risk' should be reassessed with another composite scoring system and referred to liver specialists if their risk level remains unchanged. Those with 'high risk' should be referred to specialist care. For example, NAFLD patients with NFS $<-1.455$ or FIB-4 index $<1.3$ have a 93\% negative predictive value for advanced fibrosis and cirrhosis. They can hence be safely managed in the primary care clinic. The physician should provide advice on weight loss, and encourage the patient to adopt a hypocaloric diet rich in antioxidants and fibre, as well as regular exercise. Weight loss of at least 3\%-5\% of body weight improves steatosis, and up to $10 \%$ weight loss improves necroinflammation in patients with NAFLD or non-alcoholic steatohepatitis (NASH). ${ }^{(13)}$ Aggressive control of hypertension, hyperlipidaemia and diabetes mellitus should be considered in these patients. Patients with $\mathrm{CHB}$ in the absence of necroinflammation - defined as raised alanine aminotransferase (ALT) and aspartate aminotransferase (AST) - and with low composite scores can similarly be managed in the primary care setting with six-monthly hepatocellular carcinoma surveillance ultrasonography and blood tests when indicated. ${ }^{(14,15)}$ Periodic monitoring of fibrosis assessment based on composite scores should be carried out. CHB patients with necroinflammation (raised ALT and AST) should be referred for specialist care, as they may benefit from treatment of $\mathrm{CHB}$. Table I summarises the risk stratification for common causes of CLD in Singapore based on noninvasive composite scoring systems.

\section{HOW DO I DIAGNOSE SIGNIFICANT FIBROSIS?}

Liver biopsy is the gold standard for diagnosis and staging of liver fibrosis. Other well-validated methods, known as noninvasive markers of liver fibrosis, are composite scores, direct serum biomarkers and liver imaging-based techniques (Fig. 2). These methods can be used to assess liver fibrosis in an outpatient, office setting as an alternative to liver biopsy, ${ }^{(16,17,18)}$ but come with limitations.
Table I. Noninvasive risk stratification for various liver diseases (adapted from Tapper EB and Lok AS). (10)

\begin{tabular}{|lll|}
\hline Test & Cut-off for AF & $\begin{array}{c}\text { AF probability* after } \\
\text {-ve vs. }+ \text { ve test (\%) }\end{array}$ \\
\hline Hepatitis B & $<1.00$ & 11 vs. 77 \\
\hline FIB-4 & $>2.65$ & 39 vs. 95 \\
\hline NAFLD & $<1.30$ & 27 vs. 72 \\
\hline FIB-4 & $>2.67$ & 41 vs. 94 \\
\hline NFS & $<-1.455$ & 24 vs. 73 \\
\cline { 2 - 3 } & $>0.676$ & 37 vs. 91 \\
\hline Hepatitis C & $<1.45$ & 24 vs. 80 \\
\hline FIB-4 & $>3.25$ & 43 vs. 68 \\
\hline APRI & $>1.00$ & 38 vs. 63 \\
\hline & $>1.50$ & 36 vs. 79 \\
\hline
\end{tabular}

*Advanced fibrosis (AF) is defined as F3/F4 stage. -ve: negative; +ve: positive; APRI: aspartate aminotransferase to platelet ratio index; FIB-4: Fibrosis-4 index; NAFLD: non-alcoholic fatty liver disease; NFS: NAFLD fibrosis score



Fig. 2 Diagram shows modes of staging for liver fibrosis (black arrows: invasive; white arrows: minimally invasive or noninvasive). ARFI: acoustic radiation force impulse; AST: aspartate aminotransferase; MR: magnetic resonance; NAFLD: non-alcoholic fatty liver disease; SWE: shear wave elastography; VCTE: vibration-controlled transient elastography

\section{Indirect serum markers and composite scores}

Various serum markers have been shown to correlate well with the presence or absence of liver fibrosis. These serum markers can be divided into two groups: indirect serum biomarkers with composite scores (based on simple laboratory tests and scoring systems); and direct serum biomarkers. Direct serum biomarkers reflect extracellular matrix turnover, whereas indirect markers reflect alterations in hepatic function rather than the metabolism of the hepatic extracellular matrix.

Indirect serum markers of fibrosis and composite scores include scoring systems based on patients' age, body mass index, platelet count, coagulation studies, and levels of serum albumin, AST and ALT. The most validated aetiology-specific scoring systems include FIB-4 index, APRI and NFS. These composite scoring systems are well validated in the prediction of fibrosis due to many aetiological agents (i.e. NAFLD, CHB and HCV), and have sufficient diagnostic accuracy in determining both minimal and advanced fibrosis. These simple scoring systems can assess 
the severity of liver fibrosis based on readily available laboratory parameters. As invasive reassessments may carry significant risks for patients, composite scores provide an excellent method for reassessing fibrosis over time.

Composite scoring systems are, however, not without disadvantages and limitations. While they can predict advanced and minimal fibrosis with reasonable certainty, they often fail to predict the exact grade of fibrosis. None of the composite scores are liver-specific, and the results can be influenced by comorbid conditions. For example, APRI can be falsely elevated in acute hepatitis. In addition, composite scoring systems require critical interpretation in terms of aetiology - one size does not fit all. For example, NFS can only be applied for NAFLD and is not validated for other aetiologies. While calculating composite scores, one should also keep in mind that the same test has a different cut-off depending on the aetiology.

Overall, these scores are excellent screening tests, especially to exclude advance fibrosis, with good negative predictive value. The following is a summary of these composite scoring systems and how they can be applied. Many non-patented free online calculators and websites can be used to calculate FIB-4, APRI and NFS, and provide instantaneous results once the data input fields have been filled in.

\section{Fibrosis-4 index}

The FIB-4 index is based on age, and AST, ALT and platelet levels. Although it was initially developed for fibrosis assessment in patients with HCV and HIV coinfected patients, ${ }^{(19)}$ it has been subsequently validated for use in $\mathrm{HCV}$ mono-infection, $\mathrm{CHB}$ infection ${ }^{(20)}$ and NAFLD/NASH. ${ }^{(21)}$ FIB-4 scores $<1.45$ have a negative predictive value of $90 \%$ for advanced fibrosis (meaning minimal amount of scarring) with $81 \%$ sensitivity. FIB-4 scores $>3.25$ have a positive predictive value of $65 \%$ for advanced fibrosis with $97 \%$ specificity. Patients with low fibrosis scores $(<1.45)$ can safely be followed up in the primary care clinic with yearly assessment of fibrosis scores. Any patient with an FIB-4 score $>1.45$ should undergo further evaluation to assess the stage of liver fibrosis.

\section{AST to platelet ratio}

APRI scores have been demonstrated to correlate well with fibrosis in many CLDs. ${ }^{(22,23)}$ However, apart from HCV infection, APRI values generally do not predict minimal or advanced fibrosis as well as the other scores, such as FIB-4 or NFS. Most experts recommend using APRI scores in conjunction with other noninvasive scores, especially with intermediate values for greater accuracy. An APRI score $>1$ is approximately $77 \%$ sensitive and $75 \%$ specific in predicting advanced fibrosis and cirrhosis (> F3) in patients with HCV infection.

\section{NAFLD fibrosis score}

NFS was derived by Angulo et al in a multicentre study. ${ }^{(24)}$ Its utility in excluding advanced fibrosis was validated in a separate study comparing multiple noninvasive models for fibrosis. ${ }^{(25)}$ NFS reliably predicts (with 90\% accuracy) which patients are unlikely to have advanced fibrosis on liver biopsy. Patients
Table II. Interpretation of the NAFLD fibrosis score (adapted from Angulo et al).(24)

\begin{tabular}{|ll|}
\hline NAFLD fibrosis score & Correlated fibrosis severity \\
\hline$<-1.455$ & F0-F2 \\
\hline-1.455 to 0.675 & Indeterminate score/fibrosis \\
\hline$>0.675$ & F3-F4 \\
\hline
\end{tabular}

NAFLD: non-alcoholic fatty liver disease

who have been diagnosed with NAFLD should have the first assessment of liver fibrosis based on NAFLD scores. Patients with an NFS $<-1.455$ can safely be followed up in primary care clinics. Their fibrosis score trends should be tracked over time to evaluate for progression or stabilisation. Table II outlines the interpretation of NFS.

\section{Direct serum biomarkers}

Direct serum biomarkers such as leptin, laminin, hyaluronic acid, procollagen III N-terminal peptide, tissue inhibitor of metalloproteinase- 1 and fibronectin are extensively studied for prediction of liver fibrosis. Hyaluronic acid seems to be most closely associated with fibrosis severity. ${ }^{(26)}$ Outside of clinical research and trial settings, these biomarkers are not available for routine use, as commercial kits are not readily available. Available commercial kits include enhanced liver function panel and FibroTest, which are increasingly being used to determine the extent of fibrosis.

\section{Imaging-based methods}

Imaging-based methods, including vibration-controlled transient elastography (or FibroScan $®$ ), magnetic resonance imaging, acoustic radiation force impulse imaging and shear wave elastography, typically use the principle of 'shaking the liver' with an external probe. This is based on the theory that as the liver becomes increasingly stiff, the velocity of the shear wave increases. However, these methods require specialised equipment and training, and are costly to set up, limiting their use in the primary care setting.

\section{Liver biopsy}

Liver biopsy is the gold standard test to measure liver fibrosis. ${ }^{(27)}$ However, it is an invasive procedure and not only has minor complications such as pain (20\%), but also major complications such as haemobilia, intraperitoneal bleeding $(0.5 \%),{ }^{(28)}$ and even death $(0.009 \%-0.12 \%) .{ }^{(29,30)}$ Other major drawbacks of liver biopsy include sampling errors: an adequate liver biopsy specimen can only assess a tiny fraction (1/50000) of the liver; ${ }^{(31)}$ and inter- and intraobserver variability in reporting limits its use in clinical practice. ${ }^{(32)}$ In a sentinel study involving 51 patients with NAFLD in whom two biopsy samples were obtained on the same day, $35 \%$ of the patients who had F3 fibrosis in one sample had F0 or F1 fibrosis in the other sample. ${ }^{(33)}$

WHEN SHOULD I REFER TO A SPECIALIST? $A$ vast majority of CLD patients can be managed in the outpatient primary care setting. Referral to specialists should be made for 


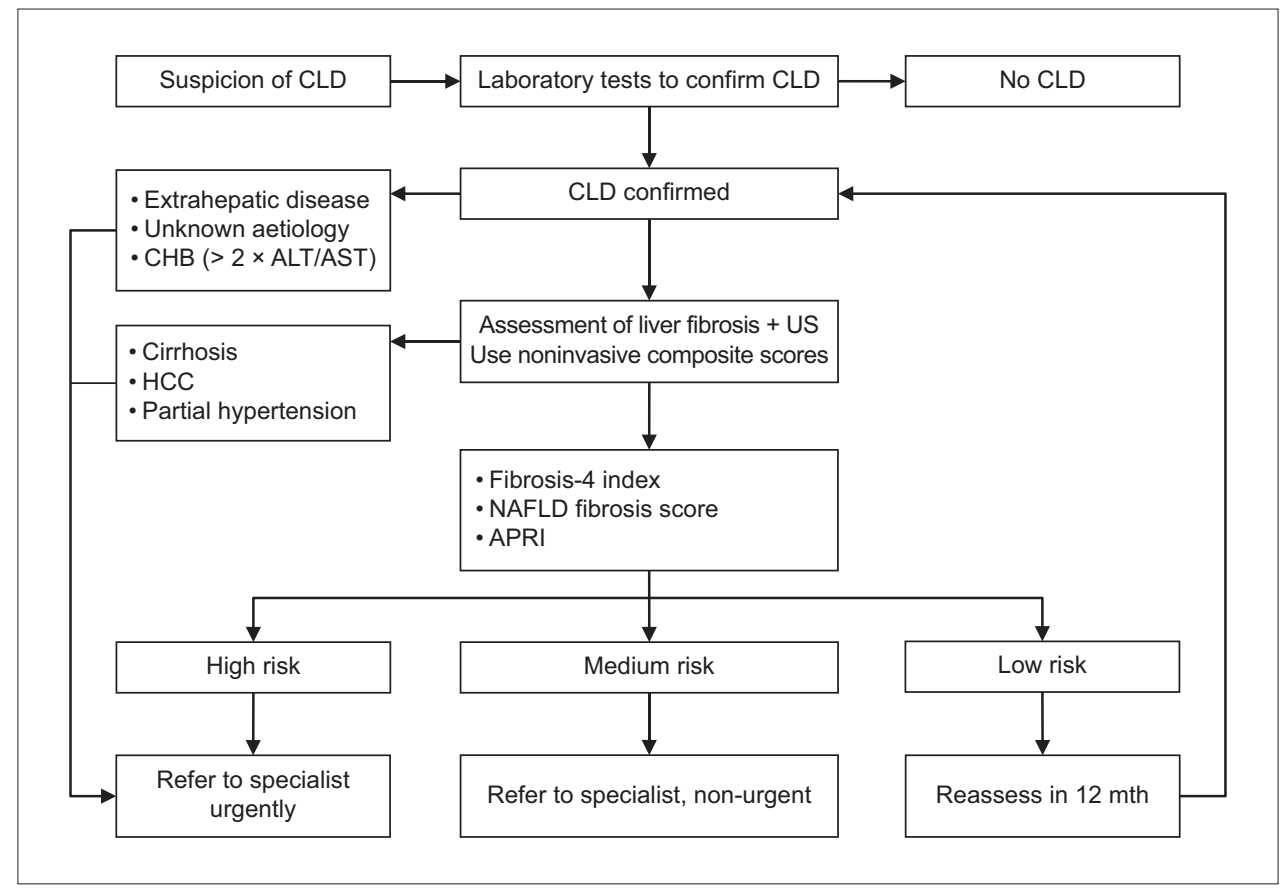

Fig. 3 Flowchart shows the noninvasive approach to clinical staging of chronic liver disease (CLD) based on composite scores. ALT: alanine aminotransferase; APRI: AST to platelet ratio index; AST: aspartate aminotransferase; CHB: chronic hepatitis B; FIB-4: Fibrosis-4 index; HCC: hepatocellular carcinoma; NAFLD: non-alcoholic fatty liver disease; US: ultrasonography

patients with signs of advanced liver disease or persistently elevated ALT and AST levels, and those without a confirmed aetiology.

Any clinical evidence of cirrhosis (i.e. ascites, jaundice, spider naevi, palmar erythema, gynaecomastia) should prompt a referral to liver specialists for further evaluation and management, as these signs are consistent with decompensated liver disease. Any radiological evidence of liver cirrhosis and portal hypertension also warrants review by liver specialists. If other causes of thrombocytopenia are ruled out, a low platelet count (typically $<150 \times 10^{3} / \mathrm{uL}$ ) is often the first biochemical marker of liver cirrhosis; any patient with persistently low platelet values should be referred for further evaluation. Patients with noninvasive composite scores (i.e. FIB-4, NFS, APRI) suggestive of advanced fibrosis ( $\geq F$ 3), or those with $\mathrm{CHB}$ infection and persistently raised ALT levels (an indication for antiviral therapy) should be referred for further management. Fig. 3 shows the noninvasive approach to clinical staging of CLD using composite scores.

\section{TAKE HOME MESSAGES}

1. CLD is becoming more prevalent as NAFLD increases rapidly due to the obesity and diabetes mellitus epidemic.

2. A high index of suspicion is essential for CLD, as most patients are asymptomatic and incidentally diagnosed.

3. Easy-to-use composite scoring systems for fibrosis assessment can identify patients who are at risk for minimal or advanced fibrosis, and should be an integral part of the decision on whether to refer to specialist care.

4. NAFLD patients with minimal or no fibrosis based on composite scores can be safely managed in primary care clinics.
5. Patients with high likelihood of advanced fibrosis based on composite scores, $\mathrm{CHB}$ with necroinflammation (raised ALT and AST) or deranged liver function tests of uncertain aetiology should be referred to a liver specialist.

ABSTRACT Liver fibrosis is a slow, insidious process involving accumulation of extracellular matrix protein in the liver. The stage of liver fibrosis in chronic liver disease (CLD) determines overall morbidity and mortality; the higher the stage, the worse the prognosis. Noninvasive composite scores can be used to determine whether patients with CLD have significant or advanced fibrosis. Patients with low composite scores can be safely followed up in primary care with periodic reassessment. Those with higher scores should be referred to a specialist. As the epidemic of diabetes mellitus, obesity and non-alcoholic fatty liver diseases is rising, CLD is becoming more prevalent. Easy-to-use fibrosis assessment composite scores can identify patients with minimal or advanced fibrosis, and should be an integral part of decision-making. Patients with cirrhosis, high composite scores, chronic hepatitis B with elevated alanine aminotransferase and aspartate aminotransferase, or deranged liver panel of uncertain aetiology should be referred to a specialist.

Keywords: chronic liver disease, fibrosis assessment, noninvasive composite scores

\section{REFERENCES}

1. Friedman SL. Evolving challenges in hepatic fibrosis. Nat Rev Gastroenterol Hepatol 2010; 7:425-36.

2. Kawada N. Evolution of hepatic fibrosis research. Hepatol Res 2011; 41:199-208.

3. Bataller R, North KE, Brenner DA. Genetic polymorphisms and the progression 
of liver fibrosis: a critical appraisal. Hepatology 2003; 37:493-503.

4. Martini A, Ceranto E, Gatta A, Angeli P, Pontisso P. Occult liver disease burden: analysis from a large general practitioners' database. United European Gastroenterol J 2017; 5:982-6.

5. Dulai PS, Singh S, Patel J, et al. Increased risk of mortality by fibrosis stage in nonalcoholic fatty liver disease: systematic review and metaanalysis. Hepatology 2017; 65:1557-65.

6. Yano $M$, Kumada $H$, Kage $M$, et al. The long-term pathological evolution of chronic hepatitis C. Hepatology 1996; 23:1334-40.

7. Younossi ZM, Stepanova M, Rafiq N, et al. Pathologic criteria for nonalcoholic steatohepatitis: interprotocol agreement and ability to predict liver-related mortality. Hepatology 2011; 53:1874-82.

8. Angulo P, Kleiner DE, Dam-Larsen S, et al. Liver fibrosis, but no other histologic features, is associated with long-term outcomes of patients with nonalcoholic fatty liver disease. Gastroenterology 2015; 149:389-97.e10.

9. Ekstedt $\mathrm{M}$, Hagström $\mathrm{H}$, Nasr $\mathrm{P}$, et al. Fibrosis stage is the strongest predicto for disease-specific mortality in NAFLD after up to 33 years of follow-up. Hepatology 2015; 61:1547-54.

10. Tapper EB, Lok AS. Use of liver imaging and biopsy in clinical practice. N Eng J Med 2017; 377:756-68

11. Tapper EB, Hunink MG, Afdhal NH, Lai M, Sengupta N. Cost-effectiveness analysis: risk stratification of nonalcoholic fatty liver disease (NAFLD) by the primary care physician using the NAFLD fibrosis score. PLoS One 2016 $11: \mathrm{e} 0147237$

12. Tapper EB, Sengupta N, Hunink MG, Afdhal NH, Lai M. Cost-effective evaluation of nonalcoholic fatty liver disease with NAFLD fibrosis score and vibration controlled transient elastography. Am J Gastroenterol 2015; 110:1298-304.

13. Yamamoto $M$, Iwasa $M$, Iwata $K$, et al. Restriction of dietary calories, fat and iron improves non-alcoholic fatty liver disease. J Gastroenterol Hepatol 2007 22:498-503

14. Terrault NA, Bzowej NH, Chang KM, et al; American Association for the Study of Liver Diseases. AASLD guidelines for treatment of chronic hepatitis B. Hepatology 2016; 63:261-83.

15. European Association for the Study of the Liver. EASL 2017 clinical practice guidelines on the management of hepatitis B virus infection. J Hepatol 2017; 67:370-98.

16. Pinzani M, Vizzutti F, Arena U, Marra F. Technology insight: noninvasive assessment of liver fibrosis by biochemical scores and elastography. Nat Clin Pract Gastroenterol Hepatol 2008; 5:95-106.

17. Martínez SM, Crespo G, Navasa M, Forns X. Noninvasive assessment of liver fibrosis. Hepatology 2011; 53:325-35
18. Castera L. Noninvasive methods to assess liver disease in patients with hepatitis B or C. Gastroenterology 2012; 142:1293-1302.e4.

19. Sterling RK, Lissen E, Clumeck N, et al; APRICOT Clinical Investigators. Development of a simple noninvasive index to predict significant fibrosis in patients with HIV/HCV coinfection. Hepatology 2006; 43:1317-25.

20. Kim BK, Kim DY, Park JY, et al. Validation of FIB-4 and comparison with other simple noninvasive indices for predicting liver fibrosis and cirrhosis in hepatitis B virus-infected patients. Liver Int 2010; 30:546-53.

21. Shah AG, Lydecker A, Murray K, et al; Nash Clinical Research Network. Comparison of noninvasive markers of fibrosis in patients with nonalcoholic fatty liver disease. Clin Gastroenterol Hepatol 2009; 7:1104-12.

22. Lin $\mathrm{ZH}$, Xin $\mathrm{YN}$, Dong $\mathrm{Q}$, et al. Performance of the aspartate aminotransferaseto-platelet ratio index for the staging of hepatitis C-related fibrosis: an updated meta-analysis. Hepatology 2011; 53:726-36.

23. Sirli R, Sporea I. Aspartate aminotransferase to platelet ratio index for the assessment of liver fibrosis severity in patients with chronic hepatitis. Hepat Mon 2011; 11:560-1.

24. Angulo P, Hui JM, Marchesini G, et al. The NAFLD fibrosis score: a noninvasive system that identifies liver fibrosis in patients with NAFLD. Hepatology 2007; 45:846-54.

25. McPherson S, Stewart SF, Henderson E, Burt AD, Day CP. Simple non-invasive fibrosis scoring systems can reliably exclude advanced fibrosis in patients with non-alcoholic fatty liver disease. Gut 2010; 59:1265-9.

26. Lydatakis H, Hager IP, Kostadelou E, et al. Non-invasive markers to predict the liver fibrosis in non-alcoholic fatty liver disease. Liver Int 2006; 26:864-71.

27. Afdhal $\mathrm{NH}$, Nunes D. Evaluation of liver fibrosis: a concise review. Am Gastroenterol 2004; 99:1160-74

28. Cadranel JF, Rufat P, Degos F. Practices of liver biopsy in France: results of a prospective nationwide survey. For the Group of Epidemiology of the French Association for the Study of the Liver (AFEF). Hepatology 2000; 32:477-81.

29. West J, Card TR. Reduced mortality rates following elective percutaneous liver biopsies. Gastroenterology 2010; 139:1230-7.

30. Terjung B, Lemnitzer I, Dumoulin FL, et al. Bleeding complications after percutaneous liver biopsy. An analysis of risk factors. Digestion 2003; 67:138-45.

31. Bedossa $P$, Dargère $D$, Paradis V. Sampling variability of liver fibrosis in chronic hepatitis C. Hepatology 2003; 38:1449-57.

32. Ter Borg $\mathrm{F}$, ten $\mathrm{K}$, Cuypers $\mathrm{H}$, et al. A survey of liver pathology in needle biopsies from HBsAg and anti-HBe positive individuals. J Clin Pathol 2000; 53:541-8.

33. Ratziu V, Charlotte F, Heurtier A, et al; LIDO Study Group. Sampling variability of liver biopsy in nonalcoholic fatty liver disease. Gastroenterology 2005; 128:1898-906 


\section{SINGAPORE MEDICAL COUNCIL CATEGORY 3B CME PROGRAMME} (Code SMJ 201812A)

1. Liver fibrosis, regardless of the underlying aetiology, is a consequence of the accumulation of extracellular matrix protein, including collagen, in the liver.

2. Environmental and not genetic factors influence the natural history of liver fibrosis.

3. The stages of liver fibrosis in chronic liver disease (CLD) define the patient's overall morbidity and mortality.

4. Noninvasive tests, especially serology-based composite score, cannot be used to determine whether patients with mild abnormalities in liver chemical values need referrals to liver specialists.

5. In patients with CLD, the assessment of liver fibrosis is not required for making therapeutic decisions and predicting outcomes.

6. The Fibrosis-4 (FIB-4) index is based on age and aspartate aminotransferase, alanine aminotransferase and platelet levels.

7. Imaging-based methods of measuring liver fibrosis are based on the theory that the stiffer the liver is, the higher the velocity of the shear wave.

8. Liver biopsy is not the gold standard test for liver fibrosis.

9. Patients with advanced fibrosis should be referred to specialist care.

10. The most common cause of liver fibrosis in Singapore is autoimmune liver disease.

11. Chronic hepatitis $C$ is more prevalent among intravenous drug users.

12. Leptin, laminin, hyaluronic acid and procollagen III $\mathrm{N}$-terminal peptide are examples of direct serum biomarkers for liver fibrosis.

13. A high index of suspicion is essential for diagnosis of CLD, as most patients are asymptomatic and diagnosed incidentally.

14. Liver fibrosis generally has an insidious onset, slowly progressing over many years, and typically takes over 20 years to develop into liver cirrhosis.

15. Liver fibrosis can be staged using composite scoring systems, liver imaging techniques, direct serum biomarkers or liver biopsy.

16. No online calculators and websites offer free calculation of FIB-4 index, APRI (aspartate aminotransferase to platelet ratio index) and NFS (non-alcoholic fatty liver disease fibrosis score).

17. Liver biopsy has complications such as bleeding, pain and even death.

18. Patients with clinical evidence of cirrhosis should be referred to liver specialists for further evaluation and management.

19. Patients with minimal or no fibrosis based on composite scores can be safely managed in primary care clinics.

20. Easy-to-use composite scoring systems for fibrosis assessment can identify patients who are at high risk for minimal or advanced fibrosis.

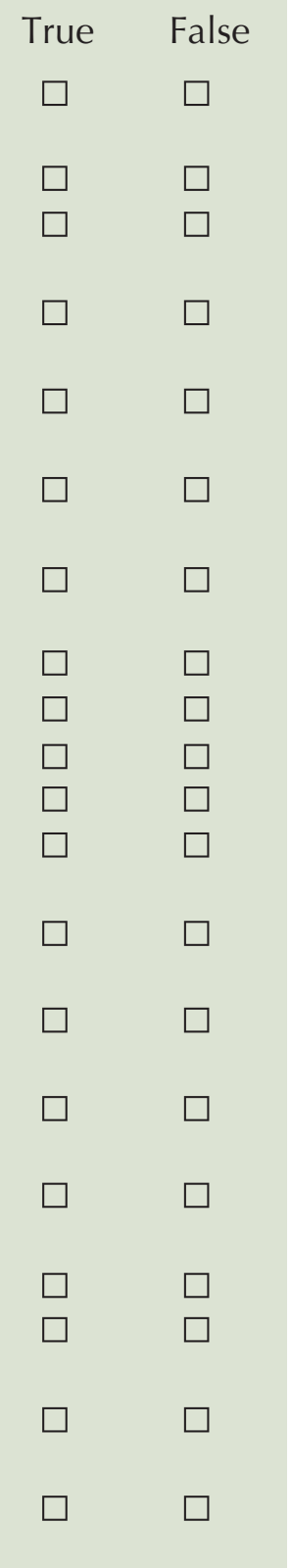

\section{Doctor's particulars:}

Name in full:

MCR no.:

Specialty:

Email:

\section{SUBMISSION INSTRUCTIONS:}

Visit the SMJ website: http://www.smj.org.sg/current-issue and select the appropriate quiz. You will be redirected to the SMA login page.

For SMA member: (1) Log in with your username and password (if you do not know your password, please click on 'Forgot your password?'). (2) Select your answers for each quiz and click 'Submit'

For non-SMA member: (1) Create an SMJ CME account, or login with your SMJ CME username and password (for returning users). (2) Make payment of SGD 21.40 (inclusive of $7 \%$ GST) via PayPal to access this month's quizzes. (3) Select your answers for each quiz and click 'Submit'.

RESULTS:

(1) Answers will be published online in the SMJ February 2019 issue. (2) The MCR numbers of successful candidates will be posted online at the SMJ website by 1 February 2019 (3) Passing mark is $60 \%$. No mark will be deducted for incorrect answers. (4) The SMJ editorial office will submit the list of successful candidates to the Singapore Medical Council. (5) One CME point is awarded for successful candidates. (6) SMC credits CME points according to the month of publication of the CME article (i.e. points awarded for a quiz published in the December 2017 issue will be credited for the month of December 2017, even if the deadline is in January 2018).

Deadline for submission (December 2018 SMJ 3B CME programme): 12 noon, 25 January 2019. 